\title{
초록
}

본 연구는 교수설계 타당화를 위하여 Toulmin(1958, 2003)의 논증구조에 기반한 교수설계모형을 제안한다. 기존의 교수설계에 사용되는 모형은 Dick과 Carey, ADDIE 모형 등이 있는데, 이 모형들은 증거를 수집하는 단계의 타당성을 확보하는 과정이 부족하다는 한계를 지니고 있다. 즉, 기존의 교수설계 모형은 요소를 구성하는 데 중점을 두기에 각 단계마다의 절차적 타당성을 확보하는 데 부족하다는 한계를 지닌다. 이 논문에서는 Toulmin의 논증구조를 이용하여 교수설계 과정의 타당화를 위한 모형을 제공하는데, “교수계획안”, “교수설계안”, “교수운영계획안”, “총괄평가 결과” 등의 네 개의 결과가 있고, 각 결과를 지지하는 논거와 논거를 지지하는 증거를 수집하는 방향으로 각 결과의 타당성을 확보한다. 또한 이 결과들을 반박하는 반박논거 및 반박증거 등을 고려함으로써 교수설계의 개선점을 구체적으로 알 수 있는 모형이다. 지지하는 증거와 반박하는 증거를 고려함으로써 교수설계의 타당성을 높일 수 있는 모형으로 $\mathrm{C}$ 대학의 교수설계 과정의 타당성을 확보하는 과정을 제시하였다.

주제어: 교수설계모형, 프로그램 사정, 논증기반타당화, 역량기반교육, $\mathrm{ADDIE}$ 


\section{1. 배경}

국내 대부분의 대학들은 최근 급감한 학령인구로 인해 상당한 문제를 접하고 있으며, 교육부는 4차 산업혁명 시대에 교육의 질 향상과 대학의 혁신을 위해 대학기본역 량진단을 시행하고 있다. 이에 대응하여 대학은 창의력, 비판적 사고력 등과 같은 학생의 역량을 향상시키기 위해 교육과정을 혁신하고 있다. 급변하는 상황에 대처하기 위해 일부 대학은 ADDIE모형이나 PDCA모형 등 교수설 계와 교육성과관리 모형을 이용하여 교육과정의 개발과 평가를 위해 체계적인 접근을 하고 있으나 기존의 교수설 계모형은 요소를 구성하는 데 중점을 두기에 각 단계마다 의 절차적 타당성을 확보하는 데 부족하다는 한계를 가진 다. 본 연구에서는 Toulmin(1958, 2003)의 논증구조에 기반하여 각 단계마다의 타당성을 확보하기 위한 절차를 다루는 포괄적인 모형을 제안하고자 한다.

역량(competency)이란 지식(knowledge), 능력(ability), 기술(skills) 등을 포괄하는 개념이다. OECD DeSeCo(2005) 에서는 a) 도구(언어나 기술 등)를 상호적으로 사용, b) 다양한 집단에서의 상호작용 및 c) 자율적 행동 등의 세 가지 역량의 범주를 정의하고 기술하고 있다. 언어와 기술 을 사용하고, 다른 집단의 구성원과 협동을 할 수 있고, 갈등을 해결하는 능력, 나아가 자신의 흥미와 권리를 찾 고, 자신의 미래에 대한 비전을 설계하는 능력 등을 역량 으로 정의한다.

출생률의 감소로 인해 현재 학령기 인구가 대학의 정원 을 채우지 못하는 상황에서 대학의 구조조정과 교육프로 그램의 혁신을 도모하기 위해 2014년부터 대학의 기본역 량을 진단하는 평가가 실시되어 왔으며, 각종 평가에서 역량을 강조하고 있다. 대학기본역량진단 3주기 편람에 따르면, 교양교육과정에서는 핵심역량(예: 사고력, 창의 력 자기개발능력 등)을, 전공교육과정에서는 전공능력을 평가하고 있으며, 이러한 평가 기준 및 시대적 요구에 따라 많은 대학들이 각각의 역량을 개발하고, 학생들의 역량을 향상시키기 위한 교육과정을 개발 - 운영하고 그 결과를 환류하고 있다.

교육운영의 일련의 과정에서 각 대학들은 교육설계모형으 로 ADDIE(Analysis, Design, Development, Implementation, Evaluation)모형을, 성과관리모형으로 PDCA(Plan, Do, Check, Act) 또는 CIPP(Context, Implementation, Process,
Product)등을 주로 사용함으로써 교육과정 설계와 성과관 리를 아우르는 모형을 사용하고 있다. 이러한 모형은 교육 과정 개발과 평가에 이르는 과정을 단순하고 체계적으로 정의하고 있기에 많은 대학에서 모형의 절차에 맞게 따르 기가 용이하다. 하지만 절차가 타당하다고 해서 타당한 결과가 나오는 것은 아니다. 즉, 절차에 맞게 교육과정을 개발했지만, 어떤 증거를 얼마나 모아야 하는지에 관한 사항은 한계점으로 남아있다. 예를 들어 $\mathrm{ADDIE}$ 모형의 분석(analysis)에서 이해관계자들로부터의 수요조사를 통 해 분석을 하지만, 누구를, 언제, 어떤 방식으로 수요조사 를 수행했는지에 관한 타당성 여부를 검증하기는 어렵다. 따라서 본 논문에서는 기존의 교육과정 개발 및 평가에 관한 모형을 수정하여 각 단계마다 교과목 개발의 타당성 을 검증하는 모형을 제공해 교육부의 기본역량진단을 대 비하고 있는 대학에 타당성이 높은 교수설계모형을 제안 하고자 한다.

\section{2. 국내 대학에서 활용하는 교수설계 모형}

\section{1 교수설계모형}

교수설계모형은 교육과정을 개발하고 사정하는 데 필 요한 구성과 절차를 제공하는 역할을 한다. 교수설계모형 에 따라 필요한 요소를 제시함으로써 교육과정 개발의 효율성을 높이는 데 유용하다. 현재 한국대학에서는 이러 한 교수설계모형을 이용하여 기본역량 진단과 관련하여 교육프로그램 개발 및 사정을 진행하고 있다. 이 섹션에서 는 한국대학이 전반적으로 이용하는 교수설계모형을 소 개하고 각 모형의 강점과 약점을 살펴봄으로써 이 논문의 후반부에 교수설계모형을 제안하고자 한다.

대학에서 대체적으로 많이 활용되는 교수설계모형으 로 Dick과 Carey 모형(Dick \& Carey, 1985; Dick, Carey \& Carey, 2004), Seels와 Richey(1994)의 ADDIE(analysis, design, development, implementation, evaluation)모형, Kemp모형(Morrison, Ross \& Kemp, 2004; Morrison, Ross, Kemp, \& Kalman, 2010), Willis(2000)의 R2D2모 형, Smith와 Ragan(1999) 교수설계모형 등이 있다. 이 모형들은 체계적(process) 접근과 체제적(system) 접근으 로 나눌 수 있다. 체계적 접근은 순서와 절차를 구성하는 
모형으로서 단계적, 선형적 설계절차를 지니고 있으며 교육목표의 효과적, 효율적 달성을 위해 수업활동을 설계 하기 위해 사전에 명세화된 절차에 기반한다. 반면 체제적 접근은 총체적인 조직을 나타내고 교육목적을 달성하기 위해 맥락적인 특성을 고려하여 융통성 있게 설계함으로 써 역동적, 비선형적, 순환적 절차를 지니고 있으며 맥락 요인이나 변인을 고려한다.

체계적 접근에 해당하는 모형으로 대표적으로 Dick과 Carey, ADDIE 모형을 들 수 있다. Dick과 Carey 모형은 투입, 과정, 산출의 논리의 기반으로 각 단계가 연결되어 있다. [그림 1]처럼, 이 모형은 교수목적 확인, 교수 분석, 학습자 분석, 교수목표 기술, 준거기반 평가도구 개발, 수업전략 개발, 수업자료 개발, 수업형성평가 실시, 교수 수정, 총괄평가 실시 등의 과정을 거친다.

교수목적 확인단계는 학습자가 학습을 마친 뒤의 성취 결과를 기술하는 과정이다. 이러한 목적을 바탕으로 교수 분석과 학습자 분석을 실시한다. 교수 분석은 교수목적 수행을 위한 하위 지식, 기술, 태도를 분석하고 교수의 순서를 정의한다. 반면 학습자 분석은 학습자의 사전지식, 학습동기, 학습능력, 학습방법의 선호도 등을 분석할뿐만 아니라 수업장소 및 시설과 이용가능한 교수매체(예, 멀 티미디어)등을 조사한다. 이러한 교수 분석과 학습자 분 석을 바탕으로 구체적인 교수목표(준거)를 기술한다. 이 후 교수목표를 달성했는지에 관한 준거기반 평가도구를 개발하고 수업전략(교수 및 학습활동의 유형과 구체적인 절차)과 수업자료(학습자용 지침서, 교사용 지침서, 매체 자료, 평가지등)를 개발하며, 교육프로그램을 수정하고 보완하기 위한 자료를 수집하기 위해 형성평가(일대일 평가, 소집단평가, 현장평가) 등을 실시한 뒤 최종완성 후 교육프로그램의 효과성과 효율성을 검증하기 위한 자 료를 수집하기 위해 총괄평가를 실시한다.
이 모형은 교수설계자의 입장에서 교육프로그램을 설 계하는 데 초점을 두었기때문에 교수자 측면에서 교육실 행과 관련된 단계가 없다. 반면, 다음에 살펴볼 $\mathrm{ADDIE}$ 모형은 “실행” 단계가 첨가되어 있고, Dick과 Carey(2004) 모형보다는 더 단순하게 구성되어 있어서 가장 많이 사용 되는 교수설계모형이다. Dick과 Carey모형과 ADDIE모 형은 상당히 유사한 점이 많고 단지 실행 단계의 차이가 있을 뿐이다.

초기 ADDIE모형은 미국 플로리다 주립대학교에서 군 사교육을 위해 고안하여, 5가지 주요 요소(Analyze, Design, Development, Implement, Evaluation)하에 여러 가지 하위 요소들로 구성되어 있다. [그림 2]에 보이듯이 순환형의 구조를 지니고 있으며, 평가가 가운데 위치하여 각 단계를 거칠때마다 평가를 할 수 있도록 개념화 되어 있다.

ADDIE모형을 이용한 교수학습설계는 단계적으로 진 행될 뿐만 아니라 동시적으로 진행되기도 하고 어느 한 단계가 오류가 발생하면 다시 전 단계로 돌아가는 유동적 인 모형이다. 이는 모형의 가운데에 평가가 존재하기 때문 이다. ADDIE모형에서 분석단계는 이해당사자로부터의

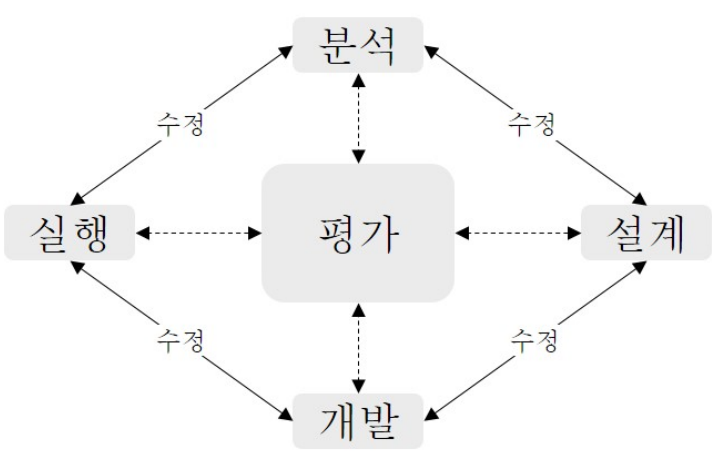

[그림 2] ADDIE 모형

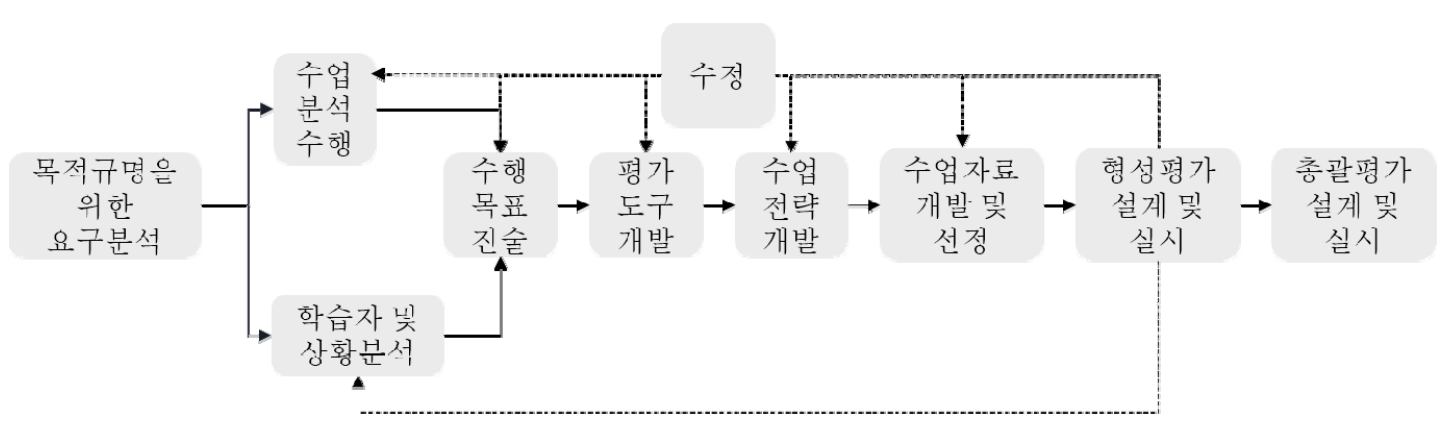

[그림 1] Dick과 Carey 모형 
요구분석, 학습자분석, 환경분석과 직무 및 과제분석을 실시한다. 이를 바탕으로 설계단계에서는 수업설계도를 제작하고 이 설계도를 바탕으로 실제 교수, 학습 프로그램 을 개발하고 제작한다. 일반적으로 이 단계에서는 프로토 타입(prototype)을 개발하여 파일럿팅을 실시하고 최종산 출물을 제작한다. 실행단계에서는 앞서 개발된 교육프로 그램을 실제 현장에 적용하고 유지 및 관리를 한다. 마지 막으로 평가는 각 단계를 동시적 - 단계적으로 평가를 함 과 동시에, 개발과 실행 이후 교수자 및 학습자를 대상으 로 프로그램의 총괄평가를 실시한다. 이처럼 체계적인 단계 설정과 유동적인 평가 단계를 도식화함으로써 교수 학습 프로그램 개발자에게 가이드라인을 제시해준다. 하 지만 $\mathrm{ADDIE}$ 모형의 단점으로는 a) 첫 단계에서 현실적이 지 못한 광범위한 '분석' 단계가 위치해 있고, b) 학습프로 그램이 준거를 충족하도록 고안되지만 학습자의 행동변 화를 알아내는 데 취약하며, c) 사후 평가는 교수학습을 향상하는 데 유용한 정보를 제공하지 못한다는 지적이 있다.

서금택과 윤용관(2019)은 한국의 주요대학에서 역량기 반교육과정 개발과 평가를 위해 사용되는 모형과 개발 사 례 등을 보여주는데, 여러 대학 중 K대학교와 SM대학교에 서 $\mathrm{ADDIE}$ 모형을 기반으로 역량중심 교육과정을 개발하 여 운영중이고, 대부분의 대학에서 ADDIE모형이 보편적 으로 사용되고 있다고 보고한다(서금택 \& 윤용관, 2019).

교수설계모형 중 단순하고 체계적이며 유용성이 높은 모형은 $\mathrm{ADDIE}$ 모형이다. 이는 한국이나 외국에서 교수설 계를 하는 데 가장 많이 사용되는 이유일 것이다. 이 논문
에서도 한국의 여러 대학이 응용하고 적용하는 ADDIE모 형에 논증구조를 적용하여 교수설계의 타당도를 높이는 모형을 3장에서 제시하고자 한다.

\section{3. 논증기반 교수설계모형}

앞서 2장에서 살펴본 교수설계모형은 절차와 요소를 구성하는 데 유용한 정보를 제공해주지만, 절차와 요소의 구성에 집중하여 각 요소의 타당성을 검증하는 데 유용하 지 못하다. 이는 교육평가분야에서 사용되는 Toulmin(1958, 2003)의 논증구조를 적용하여 교과목 설계를 위한 교수설 계의 타당성을 검증하는 데 유용한 모형을 제공함으로써 해소할 수 있다.

Toulmin 모형은 주로 교육평가에서 주로 사용되는 논 증기반타당화 모형에 Kane(1992)가 처음으로 적용하였 고, 이후로 Bachman과 Palmer(2010), Chapelle, Enright와 Jamieson(2008), Mislevy, Steinberg와 Almond(2003) 등 이 교육평가 타당화 모형에 적용하였다. Toulmin의 모형 은 일련의 논증과정을 묘사함으로써 데이터에 기반한 주 장이 타당한지 검증하는 모형이다. 주장을 하기 위해서는 이를 뒷받침하는 일련의 증거가 수반되어야 하고, 이를 반박하는 반박논거와 반박증거가 존재하면 그 주장의 타 당성은 확보될 수 없을 것이다. 예를 들어, [그림 3]과 같이 데이터에 기반한 주장을 하는 데 논거에 의해 주장이 뒷받침되고 이 논거는 증거에 의해 지지된다. 하지만 반박 논거과 반박논거를 뒷받침하는 반박증거가 존재한다면

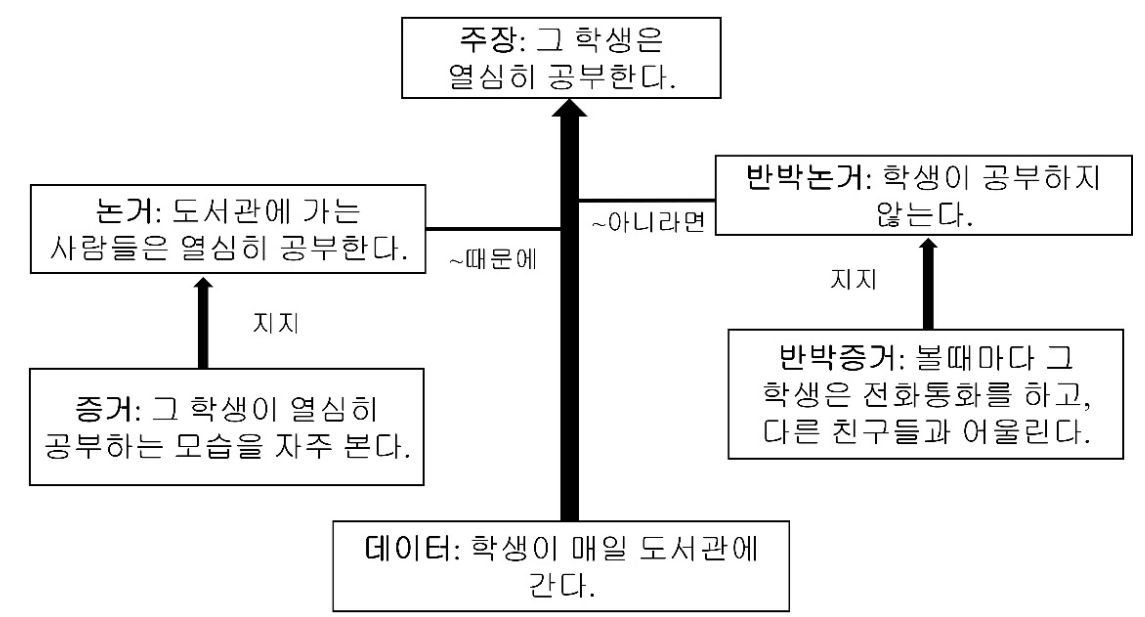

[그림 3] 논증구조(Im, Shin \& Cheng, 2019: 8) 
주장의 타당성은 확보하지 못하게 된다.

[그림 3]에 나타나듯이 데이터(학생이 매일 도서관에 간다)를 통해 “학생이 열심히 공부한다"라는 주장을 한 다. 이는 데이터를 바탕으로 주장을 이끌어 내는 추론의 과정을 묘사한 것이다. 주장의 타당성을 확보하기 위한 논거는 "도서관에 가는 사람들은 열심히 공부한다"이고, 그 논거를 뒷받침하는 증거는 "그 학생이 열심히 공부하 는 모습을 자주 본다”이다. 이러한 논거와 증거를 통해 주장은 타당성을 확보하게 된다. 하지만 그 주장은 반박논 거 "학생이 공부하지 않는다"와 그 반박논거를 뒷받침하 는 반박증거 "볼 때마다 그 학생은 전화를 하고, 다른 친구들과 어울린다"라는 증거를 통해 모형의 주장(그 학 생은 열심히 공부한다)은 타당성을 잃게 된다.

이처럼 논증기반타당화 모형은 뒷받침하거나 반박하 는 증거를 수집을 요구함으로써 데이터를 바탕으로한 주 장의 타당성을 논의하는 과정을 제공한다. 이러한 논증구 조를 교육과정설계와 평가 및 성과관리모형에 적용한다 면 체계적이고 논리적인 타당화과정을 통해 교육과정을 운영할 수 있을 것이다. [그림 3]에서 보이듯이 왼쪽에 위치한 논거와 증거는 교과목 설계를 위한 교수설계의 타당성을 확보하는 부분이고, 오른쪽의 반박논거와 반박 증거는 개선점이 될 것이다. 개선점을 명확하게 기술하여 교수설계의 타당성을 위협하는 요인들을 없애가는 과정 을 통해 교과목 설계의 타당성을 높일 수 있다. 이 논증구 조는 교육프로그램개발자나 실무자들이 개선점과 지지 하는 증거를 수집하는 데 필요한 가이드라인을 제공해주

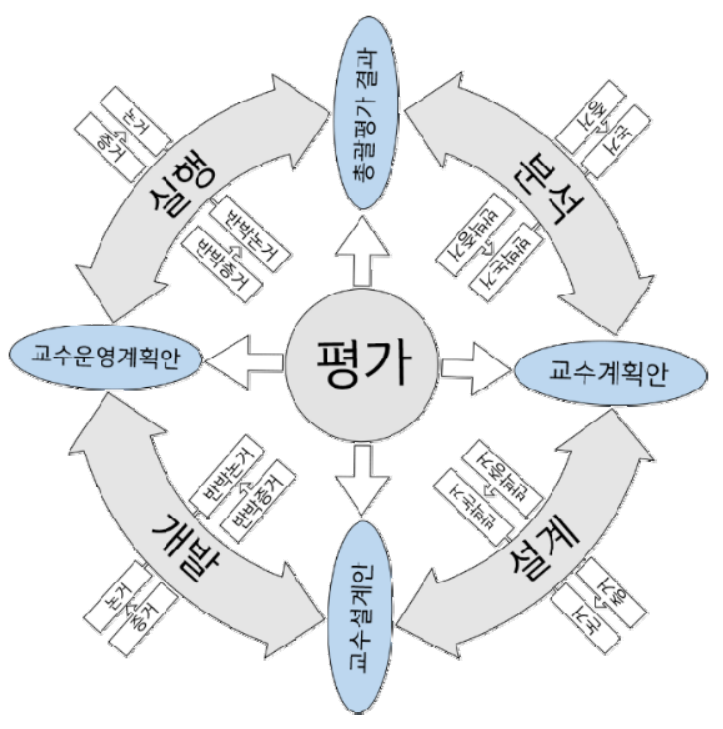

[그림 4] 물레방아 교수설계모형
는 역할을 한다.

이 논문에서 제안하는 타당화모형은 ADDIE모형에 논증 구조를 적용한다. 모형의 이름은 [그림 4]와 같이 물레방아 (waterwheel) 모형이라 명명하고 순환적이고 각 단계마다 평가(evaluation)를 구성함으로써 한 단계에서 다른 단계 로 타당성이 확보되지 않는다면 넘어갈 수 없는 형상이다.

$\mathrm{ADDIE}$ 모형의 경우 앞서 언급된 광범위한 분석단계가 위치해 있다는 비평이 있지만 실제 맥락에서 이해당사자 의 요구와 실제 상황의 분석은 교과목 설계의 타당성을 높이는 데 반드시 필요한 단계이다. 교수설계에서 주요 이해관계자로부터의 요구조사 혹은 수요조사를 하지 않 는다면 교육프로그램의 타당성을 확보하는 데 약점이 있 을 것이다. 또한 교육프로그램이 준거를 충족하도록 고안 되지만 학습자의 행동변화를 알아내는 데 취약하다는 지 적에 대해 물레방아 모형에서는 학습자의 행동변화를 알 아낼 수 있는 증거를 논증구조의 증거의 항목으로 배치함 으로써 행동변화의 증거를 수집하도록 보완할 수 있다. 사후평가가 교수학습을 향상하는 데 유용한 정보를 제공 하지 못한다는 비평에 대해 물레방아 모형에서는 논증구 조의 반박논거와 반박증거를 설정함으로써 각 단계의 형 성적 평가가 가능하다는 이점이 있다. 특히 각 분석단계마 다 타당성을 확보할 수 있는 논거와 증거를 제시하고 그에 반하는 반박논거와 반박증거를 배치함으로써 각 단계의 평가를 계속해서 진행하여 사전에 평가를 도모할 수 있고, 결과적으로 긍정적인 총괄평가 결과를 가져올 수 있을 것이다. 또한 순환구조를 가지고 있기 때문에 총괄평가 결과를 바탕으로 다시 분석추론의 단계를 거쳐야 한다. 그렇게 함으로써 교수설계의 수정을 가져올 수 있다.

또한 $\mathrm{ADDIE}$ 모형은 교수설계에 필요한 각 단계를 제 시함으로써 체계적인 일련의 과정을 거치도록 한다. 하지 만 절차를 거쳤다고 해서 타당한 결과가 나오는 것은 아니 다. 즉, $\mathrm{ADDIE}$ 모형에서 어떤 증거를 얼마나 모아야 하는 지에 관한 사항은 한계점일 것이다. 논증구조를 $\mathrm{ADDIE}$ 모형에 적용함으로써, 주장을 지지하는 증거와 반박하는 증거를 제시하도록 구조화 하여 특히 반박논거와 반박증 거를 통해 무엇이 부족하고 필요한지 명확하게 보여준다.

이 모형에 총 4 개의 추론이 있다. 분석추론은 교육과정 총괄평가의 결과로부터 부정적이거나 개선점이 발견되 었을 경우, 이를 바탕으로 교수계획안이 주장된다. 교수계 획안을 바탕으로 설계추론을 거쳐 교수설계안이 도출된 
다. 개발추론의 경우 교수설계안을 바탕으로 교수운영계 획안이 주장된다. 이 교수운영계획안을 바탕으로 긍정적 이거나 부정적인 총괄평가의 결과 및 성과가 실행추론을 거쳐 주장된다. 각 단계의 논증구조는 다음과 같다. 먼저, 분석추론 논증구조에서는 기존의 교과목에서 초래한 문 제점(데이터)을 바탕으로 분석을 거쳐 교수계획안(주장) 이 유도된다. 이러한 교수계획안을 뒷받침하는 논거는 다양한 이해관계자의 요구(예, 학생, 교직원, 졸업생 등)를 반영하고 대내외적 환경이 새로운 교육프로그램을 개발 하는 데 여건 조성이 되었을 때 교수계획안의 타당성이 확보될 수 있다. 하지만 [그림 5] 오른쪽에 위치한 반박논
거와 반박증거가 발견되었을 때 교수계획안의 타당성은 낮아진다. 이러한 반박논거와 반박증거는 교과목 개발에 서 개선안으로서 역할을 하고, 담당실무자에게 무엇이 부족하고 개선되어야 하는지에 관한 정보를 제공하는 역 할을 할 것이다.

설계추론 논증구조 단계에서는 [그림 6]과 같이 교수계 획안을 바탕으로 교과목 설계를 위한 교수설계를 하여 교수설계안이 결과물로 나오게 된다. 교육목표, 평가도 구, 워크북, 교수내용, 수업계획서 등의 구체적인 설계라 는 증거를 통해 교수설계안이 충실한 분석결과를 반영하 고 구체적이고 체계적인 설계과정을 거침으로써 교수설

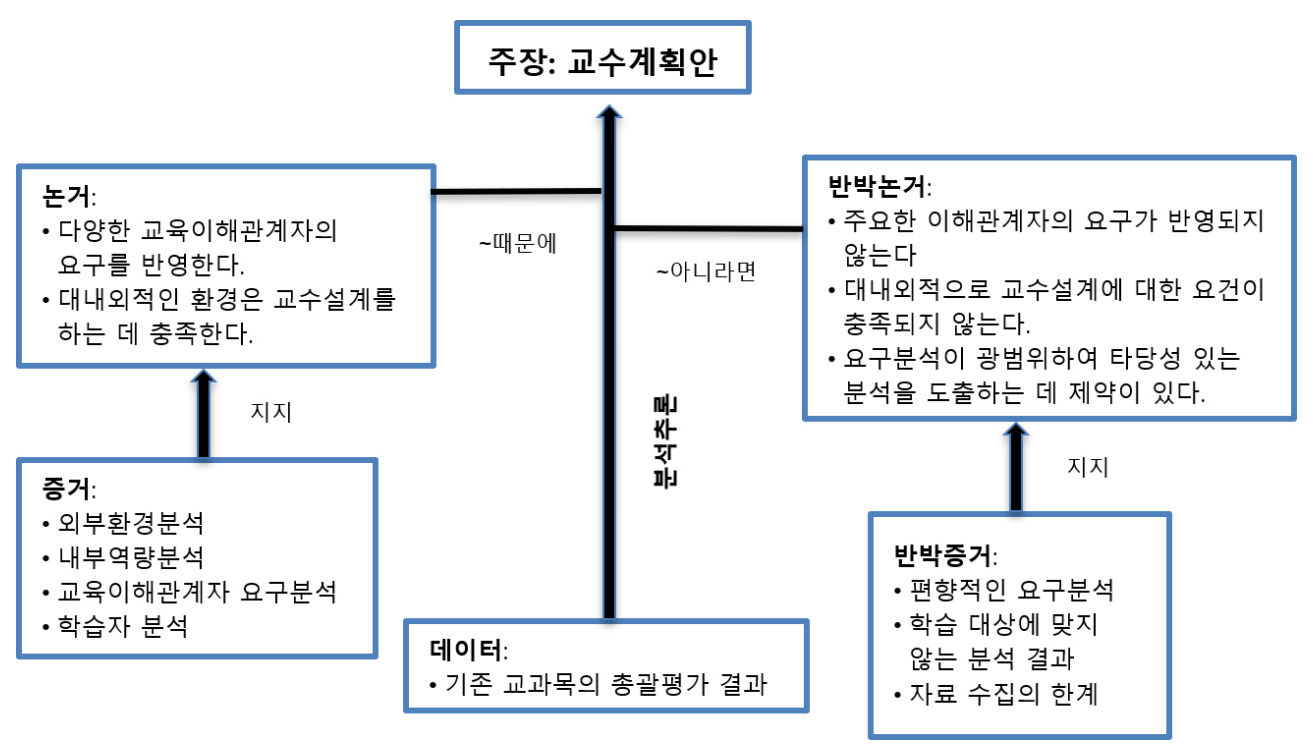

[그림 5] 분석추론 논증구조

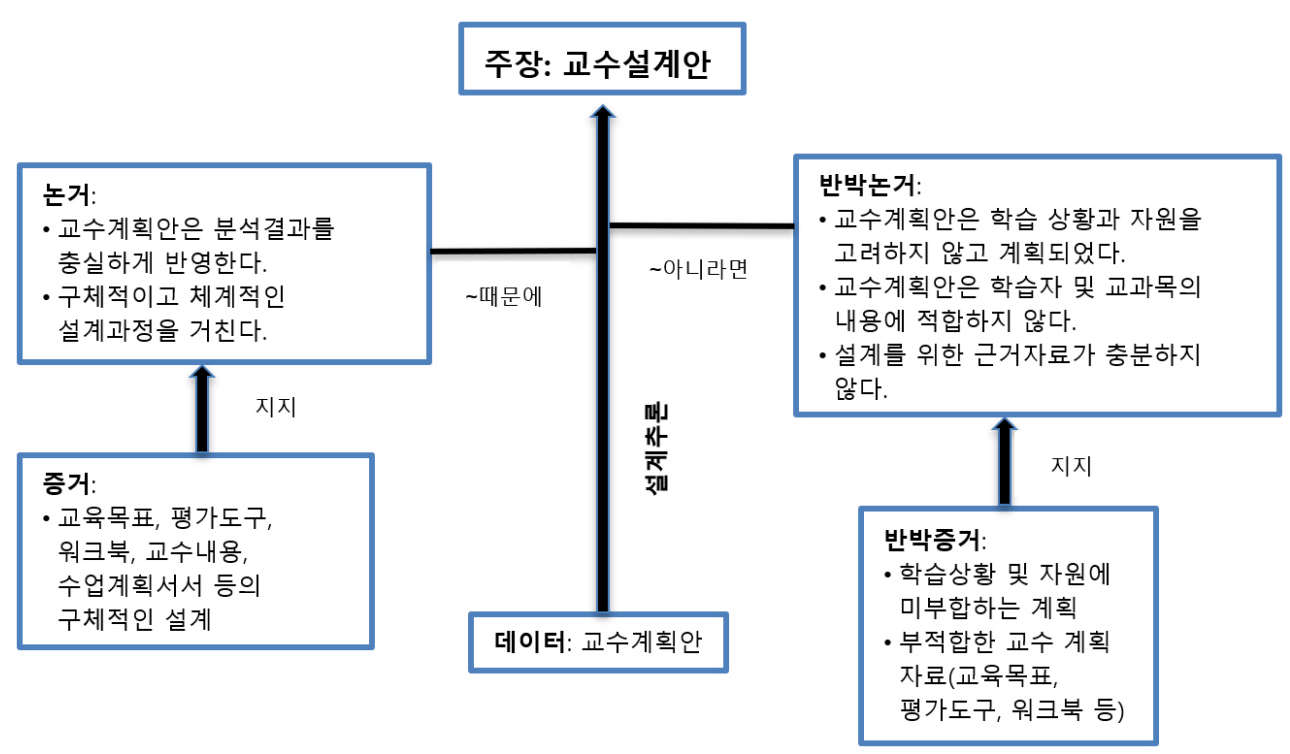

[그림 6] 설계추론 논증구조 
계안이란 주장의 타당성은 확보될 것이다. 하지만, 교수계 획안이 학습 상황과 자원을 고려하지 않은 상태에서 계획 되거나, 학습자 및 교과목의 내용에 적합하지 않고 설계를 위한 근거자료가 충분하지 않다면 교수설계안의 타당성 은 낮아지게 될 것이다.

개발추론 논증구조 단계에서는 [그림 7]과 같이 교수설 계안을 바탕으로 교수운영계획안의 결과물이 나온다. 이 교수운영계획안을 바탕으로 실행단계에서 성과 및 결과 의 결과물이 나오게 된다. 교수개발자료라는 증거를 바탕 으로 교수설계안이 증거자료를 충분히 반영하고 교수개 발 자료가 교수목표 및 내용 간 일관적이며 명료할 경우 교수운영계획안의 타당성은 확보되지만, 교수개발자료
와 교수목표 및 교수내용 간 일관성이 떨어지고, 학습 수준에 부적합한 자료가 개발되었을 경우 교수운영계획 안의 타당성은 떨어질 것이다.

실행추론 논증구조 단계에서는 [그림 8]과 같이 교수운 영계획안을 바탕으로 긍정적인 총괄평가 결과가 주장된 다. 교수운영자료(수업계획서, 수업교안, 수업 평가자료, 수업체크리스트 등)를 바탕으로 교수운영이 교수계획과 일관적이며 교수운영이 교과목의 성격에 적합할 경우 총 괄평가 결과의 타당성은 높아진다. 반면 교수운영의 적합 성 및 일관성이 떨어지고 교수운영과 교수내용의 적합도 가 미흡할 경우 총괄평가 결과의 타당성은 떨어진다.

이러한 논증구조는 각 단계의 성과관리에 있어 형성적

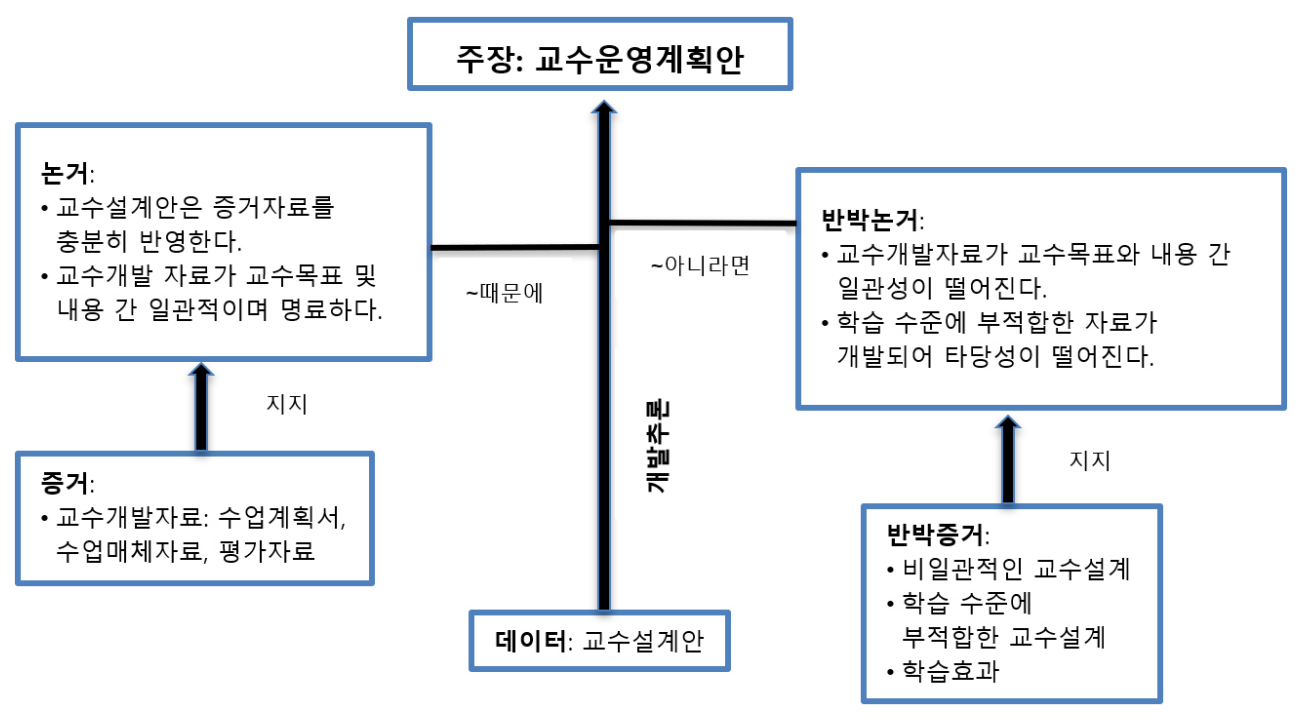

[그림 7] 개발추론 논증구조

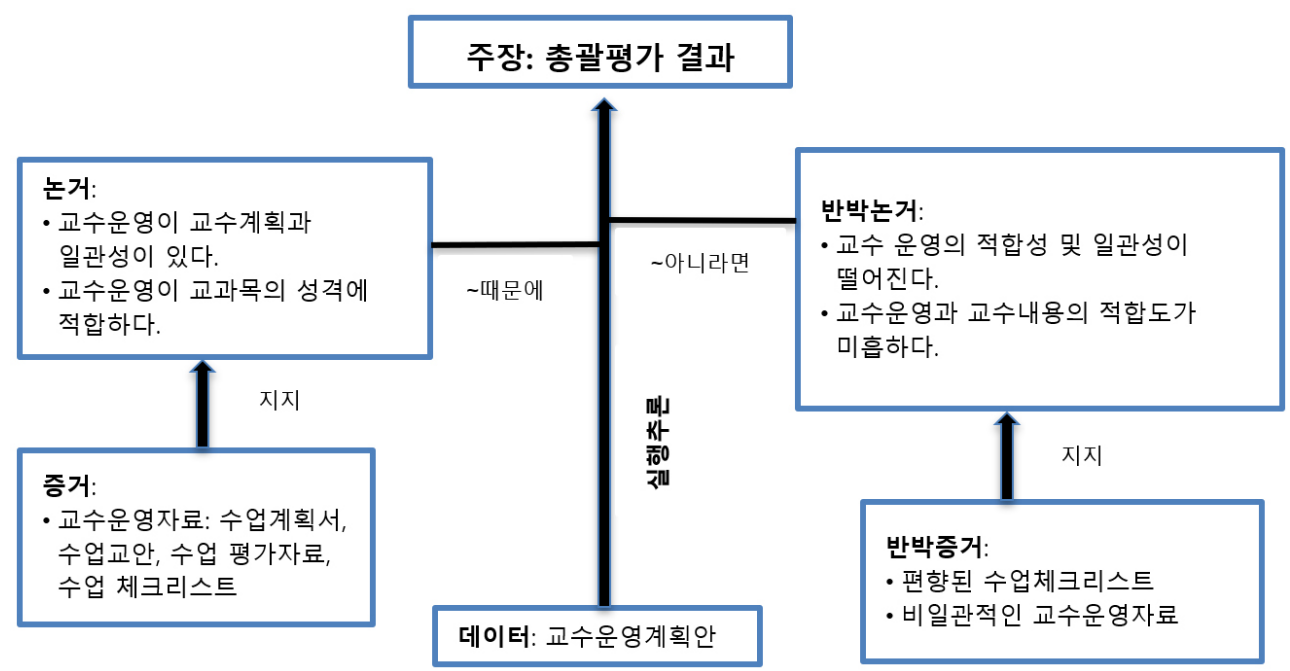

[그림 8] 실행추론 논증구조 


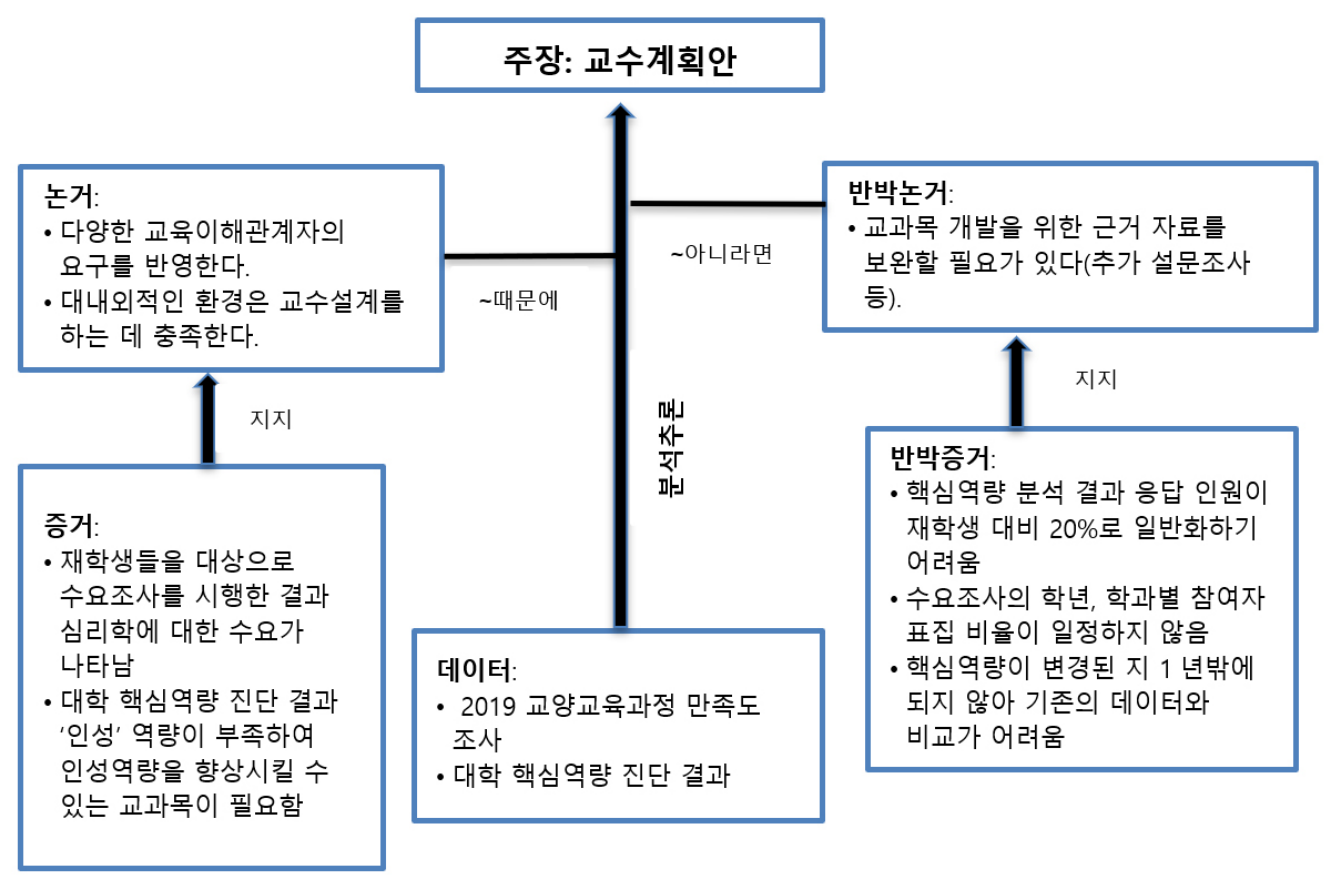

[그림 9] C대학 분석추론 논증구조

평가가 가능하고 최종실행단계에서 총괄 평가가 가능하 다. 또한 어떤 증거를 얼마나 누구로부터 얻어야 하는지에 대한 가이드라인을 제공하며, 체크리스트의 과정이 아닌, 반박논거와 반박논거의 증거가 없다는 반증주의에 기반 하여 타당성이 높은 교수설계와 성과관리를 이뤼낼 수 있다.

\section{C대학의 교수설계타당화 예시}

이 섹션에서는 교양교과목 개발 사례로써 심리학개론 의 교수설계타당화 예시를 제공한다.

[그림 9]에 제시된 것처럼 C대학에서 2019학년도 교양 교육과정 만족도 조사 결과, 교과프로그램의 다양성이 부족한 것으로 나타났고, 추가를 희망하는 교과에 대한 수요조사를 한 결과로 공동체, 글로벌, 시민의식 등을 포 함하고 있는 사회와 세계 영역의 교과목 신규 개설에 대한 요구가 나타났다. 또한 대학 핵심역량 진단결과로 '인성' 역량이 낮은 것으로 나타났고, 하위 역량인 사회관계와 자기개발 모두 낮게 나타났다. 게다가 한국교양기초교육 원 교양교육 표준안에서 인간의 본성 및 조건의 영역을 제시함에 따라, 다양한 교과목 개발, 사회와 세계에 대한 이해, 인성 역량 향상, 인간의 본성 및 조건의 영역의 사전
분석 결과에 적합하다고 판단되는 심리학 분야의 교과목 을 개설하고자 하였다. 하지만 핵심역량 분석결과 응답인 원이 재학생 대비 $20 \%$ 로 응답의 결과를 일반화하기 어렵 기 때문에 추가 설문조사 등으로 교과목 개발을 위한 근거 자료를 보완할 필요가 있다. 또한 수요조사 결과 학년, 학과별 참여자 표집 비율이 일정하지 않고 핵심역량 값이 변경된 지 1 년 밖에 되지 않아 기존의 데이터와의 비교가 어렵다는 문제점이 존재한다. 이에 기존 핵심역량인 가치 판단, 협력적소통, 자기계발이 재설정된 인성역량과 연계 됨을 확인하고, 각 영역별 교과목 수가 적고 학생들이 해당 영역에서의 만족도가 떨어진다고 응답한 자료를 토 대로 심리학개론 교과목 필요성에 대해 보완하였다.

[그림 10]에 제시된 것처럼 이전 분석추론 단계를 통해 도출된 심리학개론의 교수계획안을 바탕으로 수업계획 서를 작성한 결과, 심리학개론은 인성역량에 맵핑되어 있어 교수계획안과 일치하는 것으로 나타났다. 수업계획 서 상 교과목 개요, 학습목표, 평가도구, 교재의 기본정보 가 입력되어 있어 있으며, 인간 심리에 대한 기초 이해 과목으로 심리학의 다양한 영역을 학습한다는 교과목 개 요에 적합하게 학습심리, 사회심리, 성격심리 등 다양한 심리학의 영역을 포함하는 주차별 학습계획이 제시되었 다. 반면, 교과목의 평가 계획에서 출석, 수시고사, 기말고 사, 과제의 영역별로 구분되고 점수가 제시되어 있었으나, 


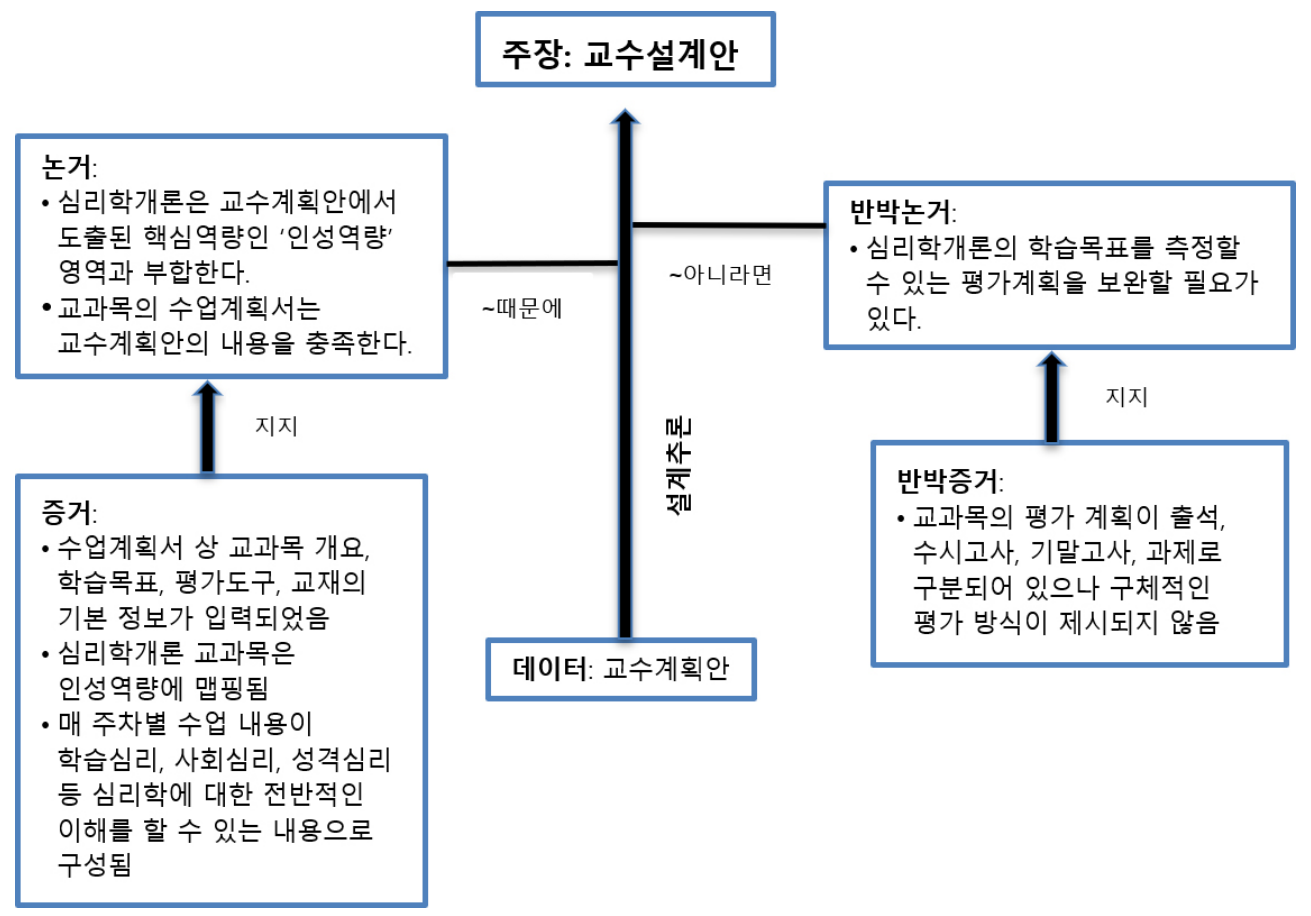

[그림 10] C대학 설계추론 논증구조

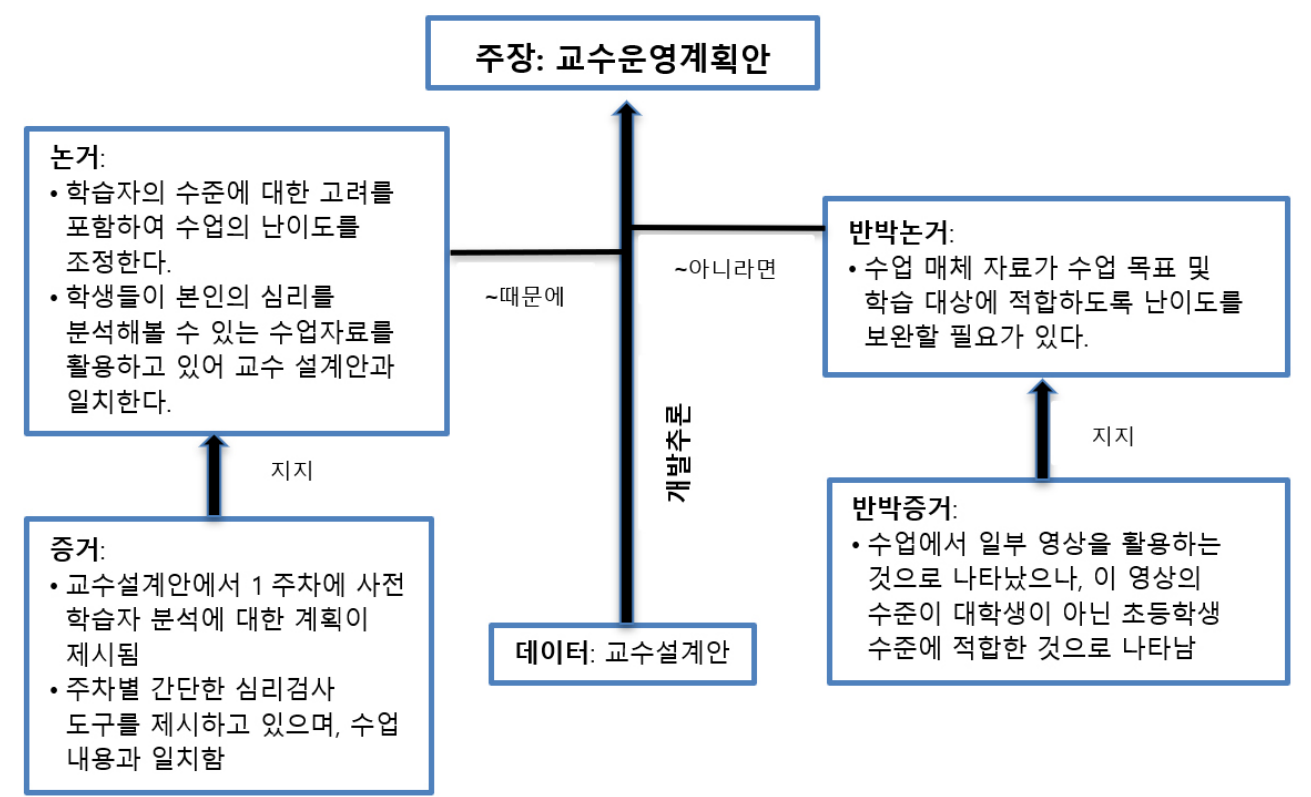

[그림 11] C대학 개발추론 논증구조

구체적인 평가의 방식은 제시되지 않았다. 이에 학습목표 에 따른 성과를 평가할 수 있는 평가계획을 보완하였다.

[그림 11]에 제시된 것처럼 교수설계안에서 1주차에 학습자 수준에 대한 분석을 실시하고자 하는 계획이 수립 되었으며, 이에 따라 학습자들이 학습목표를 달성할 수 있도록 수업의 난이도를 조절하고자 하였다. 주차별 수업 방법에도 주제에 적합한 심리검사를 진행하여, 학생들이
본인의 심리를 분석할 수 있도록 하여 교수 설계안에서 제시한 학습목표와 일치하는 것으로 나타났다. 반면, 교수 설계안에서 사용한 매체 자료 중 일부 영상이 수업을 수강 하는 대학생 수준의 자료를 제시한 것이 아닌 초등학생 수준의 자료를 제시한 것으로 확인되었다. 이에 수업 매체 자료를 대체 - 보완하여 수업목표 및 학습대상에 적합하 게 조정하였다. 


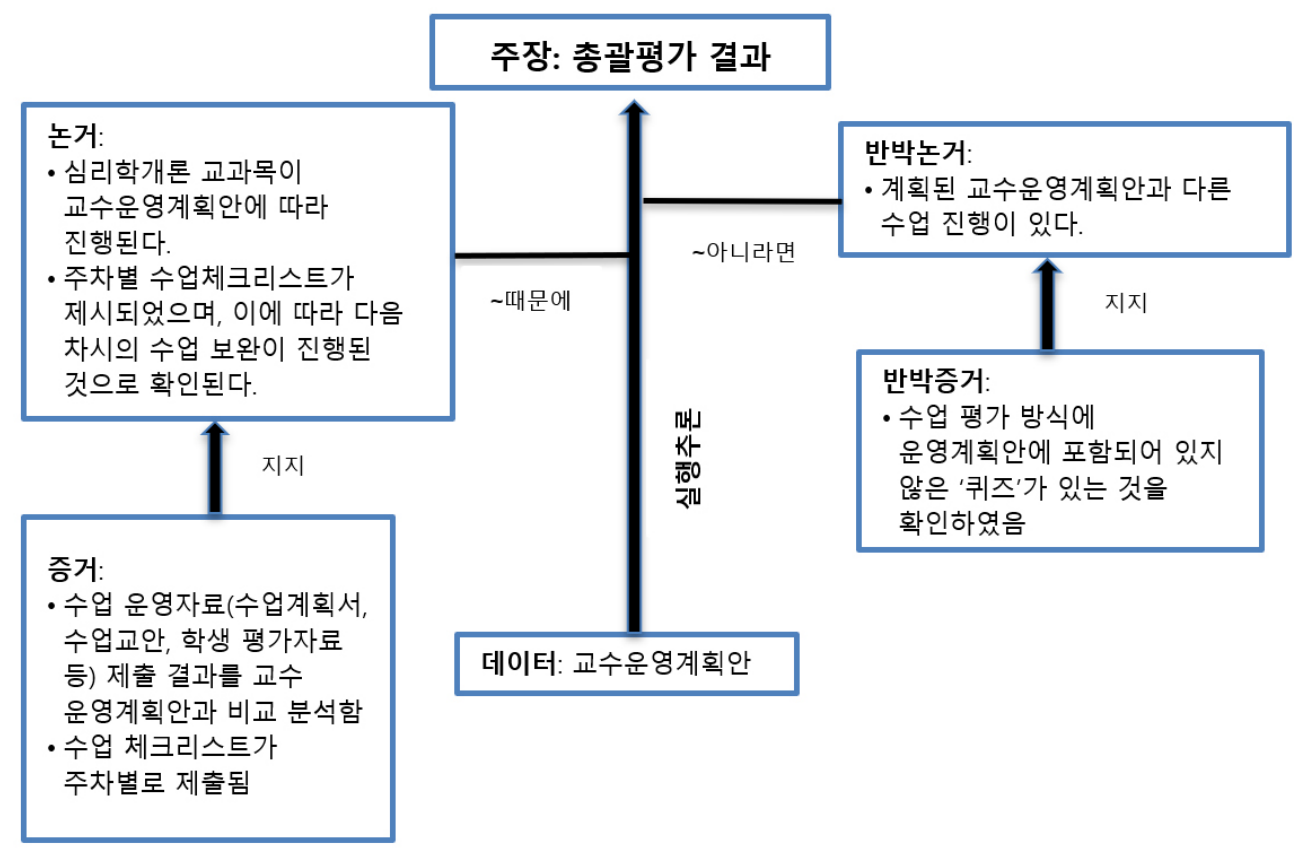

[그림 12] C대학 실행추론 논증구조

[그림 12]에 제시된 것처럼 실제 심리학개론 수업을 한학기 동안 진행하고, 수업운영자료를 교수운영계획안 과 비교 분석한 결과, 대부분의 수업 자료가 계획에 따라 진행된 것으로 확인할 수 있었다. 또한 매 주차별 수업체 크리스트를 작성하여 다음 차시의 보완에 활용한 것으로 나타났다. 그러나 수업 평가 자료에서 기존 교수설계안, 운영계획안에서 제시되지 않았던 '퀴즈'가 포함된 것을 확인할 수 있었다. 수업 체크리스트 결과, 학생들이 이해 하지 못한 수업 내용이 확인되어 퀴즈의 평가 방식을 도입 한 것으로 확인하였으나 교수운영계획안과는 불일치하 기 때문에 수업평가방식 변경 사유서를 작성하여 내용을 보완하였다.

\section{5. 결론}

본 연구에서는 Toulmin $(1958,2003)$ 의 논증구조에 기 반하여 $\mathrm{ADDIE}$ 모형의 수정을 통해 각 단계마다 교과목개 발의 타당성을 검증하는 교육프로그램 설계 및 사정 모형 을 제안하였다. 각 단계는 총괄평가의 결과에 기반하여 분석추론을 통해 교수계획안을 산출하고, 교수계획안을 기반으로 설계추론을 통해 교수설계안을, 교수설계안에 기반하여 개발 추론을 통해 교수운영계획안을, 교수운영
계획안에 기반하여 실행추론을 통해 총괄평가의 결과를 산출하는 모형을 제시하였다. Toulmin $(1958,2003)$ 의 논증구조는 주장(결과물)을 뒷받침하는 논거와 이를 지지 하는 증거를 수집하는 과정을 기술할 뿐만 아니라 반박논 거와 반박증거를 함께 수집하는 구조를 띠고 있다. 반박논 거와 반박증거는 교과목 개발에서 개선안으로서 역할을 하고, 교수설계자에게 무엇이 부족하고 개선되어야 하는 지에 관한 정보를 제공하는 역할을 할 것이다. 그리하여 타당성 높은 교수과정을 개발하는 데 도움이 될 것이다. 이러한 모형의 부재는 교수설계의 타당성을 떨어뜨리고 부정적인 결과와 성과를 가져올 것이다. 물론 절차적으로 타당하다고 해서 긍정적인 결과가 나오는 것은 아닐 것이 다. 하지만 반박논거와 반박증거의 존재는 Karl Popper의 반증주의(falsification)에 기반하여 반박하는 논거와 반박 증거가 없다는 것을 확보함으로써 교수설계의 타당성을 높이는 결과를 가져올 것이다.

본 연구에서 제안하는 모형은 각 단계를 거침으로써 단계마다 형성적 평가가 가능하고 최종 실행추론의 단계 에서 총괄평가가 가능하다는 장점이 있다. 또한 체크리스 트 접근법의 한계를 넘어 어떤 증거를 얼마나 누구로부터 얻어야 하는지에 대한 일종의 가이드라인을 제공하는 역 할을 할 것이다. 다만, 대학마다 혹은 연구자마다 각 단계 별 각기 다른 논거와 증거, 반박논거와 반박증거를 제시할 
수 있으며, 각 단계의 논증구조가 모두 동일한 형태로 적용되지 않을 수 있다는 한계가 있다. 따라서 추후 본 논증구조 모형에 따른 교과목 개발의 데이터를 축적하여 보다 일반화된 논거와 증거 기준을 보완할 필요가 있을 것이다.

\section{참고문헌}

서금택, 윤용관(2019). "대학 핵심역량과 학과 전공역량 중심의 교육과정 개발 사례”, 예술인문사회융합멀티미디어논문지 9(7), 163-173.

Bachman, L. F., \& Palmer, A. S.(2010). Language assessment in practice: Developing language assessments and justifying their use in the real world, Oxford University Press.

Chapelle, C. A., Enright, M. K., \& Jamieson, J.(Eds.)(2008). Building a validity argument for the Test of English as a Foreign Language, Routledge.

Dick, W., \& Carey, L.(1985). The systematic design of instruction (2nd ed.), Harper Collins.

Dick, W., Carey, L., \& Carey, J. O.(2004). The systematic design of instruction (6th ed.), Allyn \& Bacon.

Im, G.-H., Shin, D., \& Cheng, L.(2019). "Critical review of validation models and practices in language testing: Their limitations and future directions for validation research", Language Testing in Asia 9(1), 1-26.

Kane, M. T.(1992). "An argument-based approach to validity", Psychological Bulletin 112, 527-535.

Mislevy, R. J., Steinberg, L. S., \& Almond, R. G.(2003). "On the structure of assessment arguments", Measurement: Interdisciplinary Research and Perspectives 1, 3-62.

Morrison, G. R., Ross, S. M., \& Kemp, J. E.(2004). Design effective instruction (4th Ed.), John Wiley \& Sons.

Morrison, G. R., Ross, S. M., Kemp, J. E., \& Kalman, H.(2010). Designing effective instruction (6th Ed.), John Wiley \& Sons.

Organisation For Economic Co-Operation And Development [OECD](2005). The definition and selection of key competencies [Executive Summary].

Seels, B. B., \& Richey, R. C.(1994). Instructional technology: The definition and domains of the field, AECT.

Smith, P. L., \& Ragan, T. J.(1999). Instructional Design, John Wiley \& Sons Inc.

Toulmin, S.(1958). The uses of argument, Cambridge University Press.

Toulmin, S.(2003). The uses of argument (Updated ed.), Cambridge University Press.

Willis, J.(2000). "The maturing of constructivist instructional design: some basic principles that can guide practice", Educational Technology 40(1), 5-16. 


\section{Proposing an Instructional Design Model for Educational Program Evaluation in Tertiary Institutions}

Im, Gwan-Hyeok², Park, Ji Yoon², Shin, Hyeseong ${ }^{3}$

${ }^{1}$ Researcher, Soonchunhyang University

${ }^{2}$ Researcher, Chungnam National University

${ }^{3}$ Assistant Professor, Chungwoon University

\section{Abstract}

This study proposes an instructional design model based on Toulmin's $(1958,2003)$ argument structure to validate the instructional design. Existing models used in the instructional design include Dick and Carey, and ADDIE models. Moreover, these models have limitations in that they lack the process of ensuring validity through collecting evidence to support the instructional design. In other words, such models are known to primarily focus on what to do, and to be weak in showing progressive evidence for such outcomes.

In this study, a model for validating the instructional design process is provided using Toulmin's (1958, 2003) argument structure. The types of claims are: the "instructional plan", the "instructional design plan", the "instructional operation plan", and the "overall evaluation result". The validity is ensured through providing evidence that supports arguments". in support of the claim. In addition, it is a model in which what needs improving can be identified by considering rebuttal evidence that supports those rebuttals. By looking at the supporting and rebutting evidence in the model, the process of securing the validity of the instructional design process at University $\mathrm{C}$ is presented.

Key Words: instructional design model, program evaluation, an argument-based approach to validation, competency-based learning, ADDIE 